\title{
Outbreak Study of Foot and Mouth Disease in Relation to Vaccination and Vaccine Efficacy Assessment in Central Ethiopia
}

\author{
Beksisa Urge $^{1 *}$ and Fufa Dawo ${ }^{2}$ \\ ${ }^{1}$ Ethiopian Institute of Agricultural Research Institute (EIAR), Holeta Research center, Ethiopia \\ ${ }^{2}$ Addis Ababa University, College of Veterinary Medicine and Agriculture, Ethiopia
}

*Corresponding author: Beksisa Urge, Ethiopian Institute of Agricultural Research Institute (EIAR), Holeta Research center, Ethiopia

\section{ARTICLE INFO}

Received: 幽 April 06, 2021

Published: April 26, 2021

Citation: Beksisa Urge, Fufa Dawo. Outbreak Study of Foot and Mouth Disease in Relation to Vaccination and Vaccine Efficacy Assessment in Central Ethiopia. Biomed J Sci \& Tech Res 35(2)-2021. BJSTR. MS.ID.005685.

Keywords: Cattle; Foot and Mouth Disease; Outbreak; Vaccine

Abbreviations: DIVA: Differentiate Infected from Vaccinated Animal; FMD: Foot and Mouth Disease; FMDv: Foot and Mouth Disease Virus; NSP: Non-Structural Proteins; RCT: Randomized Controlled Trial; RR: Relative Risk; VEs: Vaccine Efficacy For Susceptibility; VEp: Vaccine Efficacy for Progression; VE: Vaccine Efficacy and Effectiveness; VEi: Vaccine Efficacy for Infectiousness

\section{ABSTRACT}

Foot and mouth disease is a viral infection affecting cloven-hoofed domestic and wild animals. An outbreak of Foot and Mouth disease in vaccinated crossbred cattle was investigated at Adea berga and Welmera districts in 2017. A total number of 90 (27.19\%) crossbred cattle were affected with FMD in the farms. The principal signs observed in clinically affected cattle were ropy salivation $(39.6 \%)$ followed by vesicle formation $(26.4 \%)$ respectively. The incidence was higher in non-vaccinated young stock $(50 \%)$ and in female cows (43\%) and the mortality proportion during the outbreak was $2.41 \%$. The overall fatality proportion in non-vaccinated crossbred animals and in vaccinated female cows was $0.43 \%$ and $0.94 \%$ respectively. In FMD endemic areas, post-infection immunity is ranged from $15-30 \%$ which indicated post-infection immunity is unlikely a component of population immunity. The past or recent infections can be detected by induced antibodies against FMDV structural proteins.

One method of serological monitoring is to identify animals in a vaccinated cattle herd that have been infected with FMD virus from vaccinated animals. Differentiating infected from vaccinated animals (DIVA) shows differences in the antibody responses produced in vaccinated animals compared to those animals are infected with FMD virus. Foot and mouth disease vaccines are purified to contain structural protein viral capsids from which majority of the viral non-structural proteins have been removed. On the other hand, the viral non-structural proteins (NSP) are expressed to elicit matching immune responses. Reduced antibody responses occurred when multiple protein conjugated vaccines sharing common epitopes have been administered simultaneously. Determining whether the current vaccines commonly used in the field could correlate with protection against new outbreaks in vaccinated and non-vaccinated animals is important.

The failure of vaccination could be due to differences between the circulating field strains and the strains included in the vaccine. Vaccine efficacy is obtained from the relative risk of infection or disease in the vaccinated animals compared to the unvaccinated individuals. When most of the population is vaccinated, some cases could not be necessarily due to vaccine failures. Cases could be excluded from the study or can be considered unvaccinated if the interval between the last dose of vaccine and the onset of symptoms is shorter than the incubation period as vaccination could lead to reduced infection in the population. Determining vaccine strain that is antigenically matched to the field strain which can provide protection against outbreaks in the field is needed. 


\section{Introduction}

Foot and mouth disease is a devastating and a highly contagious disease of cloven-hoofed animals as a result of loss of production; cost of treatments and it is the main barrier to trade of animal and animal products at local and international markets [1]. It is a virus of the genus Aphtho-virus in the family of Picronaviridae of which seven immunologically distinct serotypes (O, SAT2, SAT1, A, C, Asia 1 and SAT 3) are circulating in the wide field but multiple subtype strains have been evolved within each serotypes. Immunization with one serotype doesn't confer protection against other serotypes and fails to protect other subtypes of the same strains and this variation causes failure of the prevention and control options as infection or [2]. FMD is endemic in Ethiopia and outbreaks have been reported in different parts of the country throughout the year. The situation indicated that, four serotypes (A, O, C and SAT 2) have been identified [3]. According to [4] study, serotype 0 (73.30\%) was the most prevalent followed by A (19.50\%), SAT-2 (4.10\%), SAT-1 (1.80\%) and C (1.40\%) during outbreaks.

Besides this, [5] indicated that serotype 0 is the most prevalent strain worldwide. FMDV produced extensive vesicular lesions on the lips, tongue, gums, dental pad, feet and udder [6]. The disease is characterized by low mortality in adult cattle and massive mortality in young calves due to acute Myocarditis [7]. Authors such as [8] also reported FMD outbreak resulted in 70\% to $90 \%$ milk reduction in lactating animals. The incident of new strains and uncontrolled movement are some of the risk factors of FMD outbreak in Ethiopia [9]. Vaccination is considered as a main tool to prevent animal diseases such as Foot and mouth disease. But outbreaks of FMD have been reported after vaccination [10]. This is due to low humoral immune responses against FMDV by the locally produced vaccines and the failure of vaccine due to differences between the circulating field strains and the strains included in the vaccines [11].

Besides this, the virus could re-assort with the field strains and end up with emergence of new strain of the virus. As the duration of FMD vaccine protection is short lived, animals require revaccination twice a year. Any shift between FMDV serotypes may cause more severe outbreaks even in vaccinated animals [8]. The vaccines are DIVA (differentiate infected from vaccinated animal) capable, meaning that certain testing can distinguish between naturally infected and vaccinated animals. However, a study on the specific serotype of virus that causes outbreak is limited, so, identification of the virus strain from outbreak is very important to apply emergency vaccines [12]. Post vaccination outbreak study is important to detect newly emerging variants despite the fact that the existence of other serotypes or new virus subtypes might cause outbreak. Lack of cross protection between FMD virus serotypes as well as incomplete protection between some subtypes of FMDV affect the application of vaccine in the field.
Further, the emergence of new variant viruses makes the existing vaccine ineffective. Consequently, periodical vaccine strain selection either by in vivo methods or in vitro methods is essential to utilize efficient vaccines. The divergence between the vaccine strain and the field strains might be responsible for outbreaks of FMD in several parts of the country. Vaccination is not properly practiced under smallholder farmer's condition and disease control is complicated by the emergence of new strains which is not covered by existing vaccine. Monitoring of FMD vaccination should be carried out in cattle as well as the immunity and resistance studies of FMDV in cattle. Additionally, within serotype there are subtypes against which vaccines of the same serotype will fail to fully protect [13]. In order to achieve better control of the disease in endemic areas, it is important to investigate pre and post vaccination outbreaks to monitor the current variants of FMD virus in the field and explore the efficacy of vaccine strains which are used to combat the circulating viruses. Therefore, this study was aimed to evaluate post vaccination outbreak and explore the efficacy of FMD vaccine against circulating field strains.

\section{Materials and Methods}

The study was conducted in the dairy farms of Adea berga and Welmera areas on pre and post vaccination outbreak of Foot and Mouth Disease (FMD) in crossbred animals. During this period, a total number of 90 crossbred of cattle affected with FMD were included in this study. Foot and mouth disease outbreak was defined as the occurrence of new cases in the dairy farms [14]. Adea berga district is located in Oromia regional State, West shewa zone which is about $70 \mathrm{~km}$ from Addis Ababa. The total area of woreda is 798.35 square kilometers. It is located at 9o $12^{\prime}$ to 90 o $37^{\prime}$ latitude and $38017^{\prime}$ to 380 o $36^{\prime}$ longitude. The altitude of the area ranges from 1400 to 3500 meters above sea level. The annual average of rainfall ranges from $918 \mathrm{~mm}$ to $1368 \mathrm{~mm}$ while the minimum and maximum temperature reaches $10{ }^{\circ} \mathrm{C}$ and $25^{\circ} \mathrm{C}$ respectively. The farming system of the area is mixed type where crop production and livestock rearing are done side by side [15]. Welmera district is $40 \mathrm{~km}$ away from the capital city, Addis Ababa and the area is situated at 9o04'-9o13' N latitude and 38029'-38o39' E longitude. The average altitude of the area ranges from 2200-2500 meter above sea level. The district is known by crop-livestock mixed farming system is a common practice. The rainfall pattern of the district is bimodal, with a short rainy period from February to April and a long rainy season from mid-June to September. The annual temperature and rainfall ranges from $18{ }^{\circ} \mathrm{C}$ to $24{ }^{\circ} \mathrm{C}$ and 1000 to $1100 \mathrm{~mm}$, respectively.

\section{Foot and Mouth Disease Infected Dairy Cows Kept by Farmers}

Cattle that had manifested clinical signs of the disease and death cases in the farms were included in the study. Contributing 
factors such as age, and sex categories were considered during outbreak assessment.

\section{Outbreak Assessment and Sample Size}

Clinically and acutely sick cattle were clinically examined in the affected farms and all the visible clinical signs and parameters were recorded so as to know the status of infection in the respective farms. About 331 vaccinated-cattle and revaccinated in 2017 were used during the outbreak assessment in welmera and Adea berga districts. About 289 animals were vaccinated against FMD infection and 42 animal remains non-vaccinated. The number of sick and death animals in the farms were recorded and the proportion of clinically infected and at risks, vaccinated and infected as well as non-vaccinated and infected were determined accordingly.

\section{Vaccination, Outbreak and Vaccine Efficacy Assessments}

The farms were subsequently monitored for any disease incidence and development of clinical symptoms with special emphasis to mouth cavity and inter-digital space lesions [16]. The farm monitoring data and observations were used as baseline category of animals as vaccinated, non-vaccinated and revaccinated groups. Dairy cows that acutely exhibited clinical signs of the disease such as foamy salivation were diagnosed as well as severely infected cows that had manifested oral and pedal lesions, scrapings and healing lesions were also identified in each respective farms. The inclusion criteria in the farms are the occurrence of clinical cases and cattle that experienced the disease and clinical signs in the outbreak farms. Cattle in the same farms without any clinical syndromes excluded accordingly. The occurrence of outbreak in the farms after vaccination was checked in the farms. Vaccine assessment was performed in response to vaccination, and its protection level.

\section{Data Analysis}

The data generated from outbreak data and other field assessments were recorded and coded using Microsoft Excel spreadsheet and analyzed using STATA version 13 for Windows (Stata Corp. College Station, TX, USA). The results were tabulated and interpreted accordingly.

\section{Results}

Out Of the 331 animals observed and examined at Adea berga and Welmera districts, 90 (27.19\%) of cattle manifested clinical signs of foot and mouth disease (Table 1). The observed clinical signs in sick cattle were profuse salivation (39.6\%), vesicle on oral cavity $(26.4 \%)$, inter digital vesicles (18.8\%), and lameness $(7.5 \%)$ (Figure 1). The log odd of survival in FMD vaccinated animal is increased by 4.39 as compared to non-vaccinated groups. Foot and mouth disease infection is higher in young calves and adult cattle are more protected to infection (Table 2).
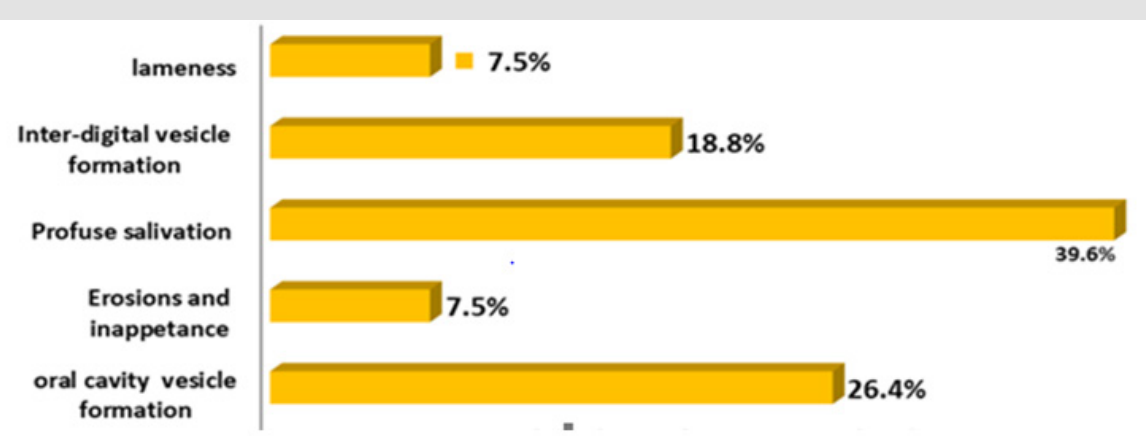

Figure 1: Clinical signs observed during FMD outbreak.

Table 1: Summary of FMD outbreaks in the respective districts.

\begin{tabular}{|c|c|c|c|}
\hline Infected Farms & No. of Animals Examined & Number of Animals Affected & No. of Death Occurred \\
\hline Adea Berga & 201 & 60 & 5 \\
\hline Welmera & 121 & 30 & 3 \\
\hline Total & 331 & $90(27.19 \%)$ & $8(2.41 \%)$ \\
\hline
\end{tabular}

Table 2: FMD outbreak linear regression analysis.

\begin{tabular}{|c|c|c|c|c|c|c|}
\hline Survival & Coefficient & Standard Error & $\mathbf{z}$ & P>[t] & \multicolumn{2}{|c|}{ [95\% Conf. Interval] } \\
\hline Age & & & & & \\
\hline Adult* & 0.7982656 & 0.1929834 & 3.06 & 0.002 & 0.328029 & 1.4725 \\
\hline Vaccine & 4.39 & 1.49 & 2.9 & 0.03 & 1.47 & 7.310 \\
\hline Yes * & -2.533172 & .894455 & -6.58 & 0.000 & $000-2.81029$ & -1.6560 \\
\hline
\end{tabular}




\begin{tabular}{|c|c|c|c|c|c|c|}
\hline sex & & & & & \\
\hline Male* & 0.3621 & 0.1952 & 0.0641 & 0.003 & 0.4731 & 1.0254 \\
\hline Cons & 0.5542657 & 0.22393 & 2.48 & 0.013 & 0.115379 & 0.99315 \\
\hline
\end{tabular}

\section{Outbreak of FMD in Vaccinated and Non-Vaccinated Animals \\ Survival of Cattle in Relation to Post Vaccination Outbreak}

Within the non-vaccinated young stock and females, the mortality proportion was $(0.5 \%)$ and $(0.43 \%)$ respectively while within the vaccinated groups, the mortality proportion in females was (0.94\%) (Table 3).

Following post vaccination outbreak in the farms, vaccinated cattle group showed $100 \%$ survival rate and the survival percentage in unvaccinated groups was $97.68 \%$. About $2.32 \%$ of the unvaccinated groups died after showing typical clinical signs of FMD (Tables 3-5).

Table 3: Mortality proportion in relation to age and sex of animals.

\begin{tabular}{|c|c|c|c|c|c|c|c|}
\hline \multirow{2}{*}{ Vaccination Status } & \multirow{2}{*}{$\begin{array}{l}\mathrm{N} \\
\%\end{array}$} & \multicolumn{2}{|c|}{ Age } & \multicolumn{2}{|c|}{ Sex } & \multicolumn{2}{|c|}{ outcome } \\
\hline & & Adult & young & Male & Female & Survival & Death \\
\hline \multirow{2}{*}{ Non-vaccinated } & $\mathrm{N}$ & 0 & 21 & 3 & 18 & 39 & 3 \\
\hline & $\%$ & 0 & 0.5 & 0.071 & 0.43 & 0.928 & 0.071 \\
\hline \multirow{2}{*}{ Vaccinated } & $\mathrm{N}$ & 240 & 49 & 18 & 271 & 289 & 0 \\
\hline & $\%$ & 0.83 & 0.17 & 0.06 & 0.94 & $318(0.99)$ & 0.009 \\
\hline
\end{tabular}

Table 4: Fatality proportion in relation to age and sex groups.

\begin{tabular}{|c|c|c|c|c|c|}
\hline Groups & Adult & young & Male & Female & Total \\
\hline FMD vaccinated (\%) & $240(0.83)$ & $49(0.17)$ & $18(0.06)$ & $271(0.94)$ & 289 \\
\hline FMD non-vaccinated (\%) & $0(0.00)$ & $21(0.5)$ & $3(0.071)$ & $18(0.43)$ & 42 \\
\hline Fisheries significant test & 0.021 & 0.001 & 0.347 & 0.000 & \\
\hline
\end{tabular}

Table 5: Cattle survived in relation to post vaccination outbreak of FMD.

\begin{tabular}{|c|c|c|c|}
\hline Categories & No. Examined & No. Died & No. Alive \\
\hline Unvaccinated calves & 12 & 1 & 11 \\
\hline Vaccinated group & 519 & 0 & 519 \\
\hline Total & 531 & 1 & 530 \\
\hline
\end{tabular}

\section{Vaccine Efficacy Assessment}

Some strains are more immunogenic than others. Vaccine efficacy is tested in vaccinated animals by challenge testing with live FMD reference viruses, distributed by the World Reference Laboratory or other FAO/OIE Reference Laboratories.

\section{Post-vaccination Assessment of Immunity at Population Level}

The overall population immunity is the proportion of animals with immunity in the whole population susceptible to infection, or vaccine that has been targeted for FMD control. This immunity occurs when large part of the population becomes immune to a virus through vaccination or infection. This is resulted from vaccine coverage and the proportion of animals responded to immunization, other sources of immunity, namely infection, earlier vaccination or maternally derived antibodies. In areas where FMD control is common and endemic, significant levels of post-infection immunity is ranged from $15-30 \%$ or beyond.

\section{Post Vaccination Reaction}

Out of 30 cattle considered for immunity assessment, a subset of 24 animals were appropriate for vaccination in the population. The analysis showed that among 24 cattle, 20 were vaccinated and the vaccine coverage was $83 \%(\mathrm{~N}=24)$, and 14 cattle produced antibodies against FMD infection, and the vaccinated population immunity was $70 \%(\mathrm{~N}=20)$. The vaccinated cattle in the entire population $(\mathrm{N}=30)$ were $67 \%(\mathrm{~N}=20)$ and the overall population immunity was $47 \%(\mathrm{~N}=14)$ (Figures $2 \& 3)$. 


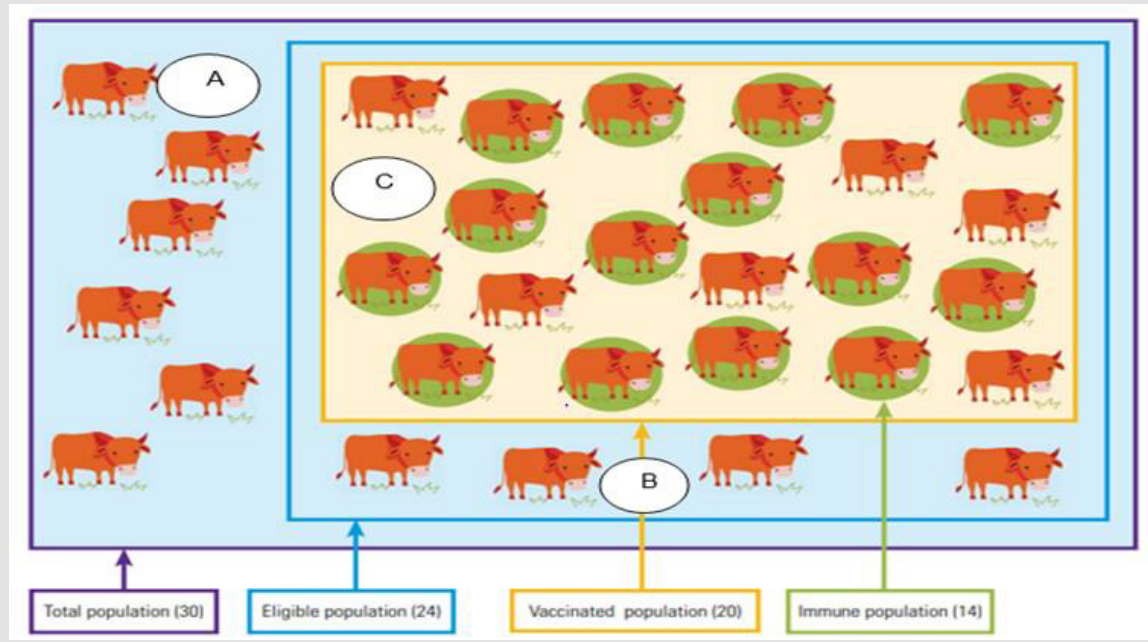

Figure 2: Non-vaccinated and vaccinated components of the population, Source: www.veterinaryworld.org (2009).

\section{Vaccinated}

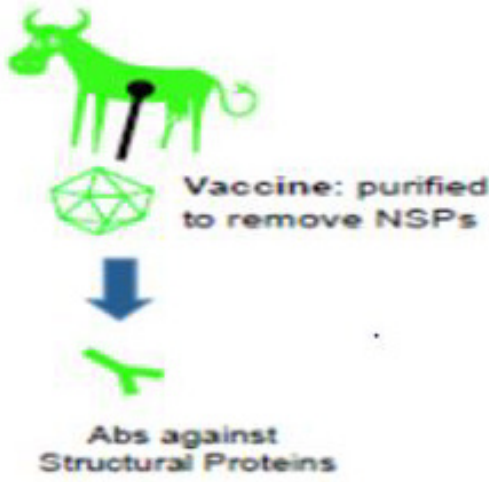

\section{Vaccinated and infected}

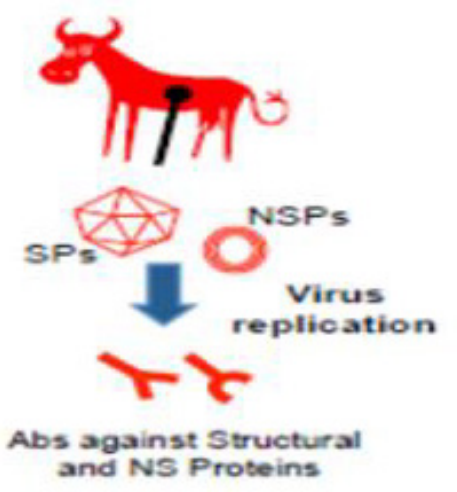

Figure 3: DIVA Principles. Source: (Ludi, et al. [18]).

\section{Immunological Study}

Foot and mouth disease virus is diagnosed by specific antibody response [17]. Serological tests are used to detect the immunity of animals exposed to foot and mouth disease virus (FMDv) or vaccines. Past or recent infection can be detected by antibodies against FMDv structural proteins. One method of serological monitoring is to identify animals in a vaccinated cattle herd that have been infected with FMD virus. DIVA (differentiating infected from vaccinated animals) principal exploits differences in the antibody responses produced in vaccinated animals compared to those animals naturally infected with FMD virus (whether they have been vaccinated or not). The quality of FMDV vaccines are purified to contain structural protein viral capsids from which majority of the FMD viral non-structural proteins have been removed. Natural infections of FMD viral non-structural proteins (NSP) are expressed to elicit matching immune response that can be diagnosed by serological principles [18].

\section{Randomized Control Trial}

The protective efficacy of vaccine and its adverse effect is evaluated by double blind randomized controlled trial (RCT). It remains the golden standard for the initial evaluation of the safety and efficacy of vaccine. Once RCT of a vaccine has been conducted and the vaccine has been shown to be efficacious, further trials can be conducted after the vaccine has been introduced into the field use. The groups or herd being evaluated or compared could be individuals or the population of animals [19].

\section{Observational and Clinical Studies}

Observational studies, direct (cohort) and indirect (casecontrol), is used to determine whether the persisting disease is the result of vaccine failure or failure to vaccinate. The allocation is nonrandom and there is potential for bias and confounding which could be controlled. The case control approach has been widely used to assess disease risks associated with non-interventional exposures, 
but with rare cases in the context of vaccine evaluation [20]. It is used to determine whether the outbreak of disease was due to vaccine efficacy or other cases and used to identify causes of the new cases. As new vaccines are introduced, booster doses are added and the timings of doses changed, clinical evaluation of vaccine efficacy is important. The analysis in case control studies employ odds ratios, conditional or unconditional logistic regression, if other variables need to be controlled [21]. Vaccine efficacy and effectiveness (VE) are explained as one minus some measure of Relative Risk (RR) in the vaccinated group compared with unvaccinated group that is VE $=1-\mathrm{RR}$. The percentage reduction in the disease condition among vaccinated subjects is attributed to vaccination [22].

\section{Susceptibility, Infection and Progression}

For infectious agents with short incubation periods, disease is used as the outcome of interest in vaccine trials rather than infection. The disease development depends on infectious source and its interaction with host that subsequently results in infection. Studies with either infection or disease as outcomes are used to measure vaccine efficacy for susceptibility (VEs). Another measure of effect to evaluate the degree of protection once the animals has become infected is vaccine efficacy for progression (VEp) with infectious agents. Vaccine efficacy for progression is conditional effect on the animals already infected, so the progression within infected individuals is important. A vaccinated and infected is less infectious to other susceptible or be infectious for a shorter period of time. The vaccine efficacy for infectiousness (VEi) is a vaccine that reduces infectiousness that could have important health consequences. This small transmission unit could be used to evaluate VEs.

The exposure to infectious agent in the vaccinated and unvaccinated groups, the estimate of VEs are obtained from the relative risk of infection or disease in the vaccinated individuals compared to the unvaccinated individuals. VEs $=1-\mathrm{RR}$ (vaccinated) / RR (unvaccinated), VEs $=1-\mathrm{q}, \mathrm{q}=$ transmission probability among vaccinated groups. Differences in transmission intensity, exposure to infection and pre-existing partial immunity and heterogeneities across herd causes different VEs estimates [23]. Vaccine effectiveness (VE) refers to vaccine protection achievement in the field. This may differ from vaccine efficacy, which refers to protection under ideal conditions. Vaccine protection in the field may differ from protection achieved under ideal conditions resulting from poor cold chain and shelf-life requirements. Vaccine effectiveness is calculated by comparing incidence of disease or infection in vaccinated animals with incidence in unvaccinated animals that were exposed to similar level of virus using the, VE= $\mathrm{RU}-\mathrm{RV} / \mathrm{RU}$, where $\mathrm{RU}$ is the incidence risk or rate in the unvaccinated population, and $\mathrm{RV}$ is the incidence in those vaccinated.

Therefore, VE (Percentage) is 1 - RV/RU in the field studies. When most of the population is vaccinated, rare cases emerge, and this could not be necessarily indicative of a declining vaccine effectiveness or efficacy. Cases can be excluded from the study or can be considered unvaccinated if the interval between the last dose of vaccine and the onset of symptoms is shorter than the incubation period. Reduced infectiousness could play a role in the transmission dynamics in populations that are nearly $100 \%$ vaccinated [20].

\section{Vaccine Matching and Vaccine Strain Selection}

The immunity to FMDV is serotype specific, and even within serotypes cross protection between strains is incomplete. Vaccine strain selection is the method of incorporating one or more vaccine strains that are able to induce protective immunity against infection from one or more circulating virus strains. A highly potent vaccine may cross-protect against a wide range of divergent strains and give relatively long-lasting immunity after a single dose. In contrast, a vaccine with a low potency will induce an antigenically narrow and short-lived protection. The protective level of induced immunity depends upon potency of the vaccine, the antigenic match between the vaccine strain and the field strain and the vaccination schedule [24].

\section{Multiple Strains and Combination Vaccines}

For infectious diseases related with a basket of strains, cross reactivity is important. Memory cells are more cross reactive than other immune cells, so that stimulation of low specificity play an important role in maintaining memory cells 23]. Widespread vaccination could allow the expansion of non-vaccine sero-types that had been less important before vaccination on the existing strains. There are immunologic mechanisms by which serologic responses to antigens in combination vaccines may differ from those obtained with separate administration of the components [25]. Antibody titers to some live virus may be lower when administered in a combination vaccine than when administered separately. Poor antibody responses is associated with multiple protein conjugated vaccines sharing common epitopes that have been administered simultaneously [26].

Eradication of Foot and mouth disease (FMD) is not possible by vaccination alone since the currently manufactured vaccines against FMD are not $100 \%$ efficacious. This sis also due to the existence of antigenically distinct types, sub types within types and emergence of antigenically variant strains of FMD virus (picorna virus with positive sense RNA) caused by mutation. Seroconversion rates (percentage of vaccines achieving a previously established protective level of antibody) need to be considered in the immunologic evaluation of combination of vaccines [27].

\section{Discussion}

Foot and mouth disease is a highly virulent disease of ruminants which can devastate the entire herd in short period of time. This was justified by frequent outbreaks affecting several cattle herds in several areas. Antibodies due to vaccination induce structural 
proteins whereas infection induces either SP or NSP antibodies. This could be due to non-immuned cattle in the vaccinated population despite being eligible late pregnancy, minimum age, potency of vaccine, application of vaccination, shelf life of vaccine and cold chain. The primary site of infection and replication of FMD is in the mucosa of the pharynx. The virus may also enter through skin lesions or the alimentary tract and the virus replicates in the epithelium of the mouth, muzzle, teats, feet, and areas of damaged skin. The disease is clinically manifested by fever and vesicular eruption in the mouth, nose, muzzle, foot, teats and other hairless soft areas of the body and it is extremely fatal in calves. The expected protection percentage (EPP) is used as reference to protective vaccine induced responses.

The EPP related to antibody titer is measured at 60 day post vaccinated $(\mathrm{dpv})$, with the percentages of protection achieved for the same groups of animals after in vivo challenge experiments performed at $90 \mathrm{dpv}$ [28]. The acutely infected cattle showed excessive foamy salivation, erosion and ulceration in the mouth and between hooves as well as blisters in the oral cavity. The relevant clinical syndromes identified during outbreaks were oral and pedal lesions. The death of foot and mouth infected calves is due to necrosis of cardiac muscle and cardiac failure [29]. More than 50\% of ruminants that recover from illness and those that are vaccinated and have been exposed to virus can carry virus particles in the pharyngeal region up to 3.5 years in cattle and 9 months in sheep [30]. The oral and pedal lesions observed in the infected and dead cattle were typical of FMD infection.

Vaccination is an important component that could reduce the burden of FMD and block circulation of the virus. The disease is mainly controlled by vaccination and is used to reduce morbidity and mortality [23]. The level of neutralizing antibodies that correlate with protection are based on experimental challenge studies giving high levels of virus at a single time point through inoculation routes. Natural exposure is made likely through multiple routes, at variable levels and for variable periods of time under field conditions and different levels of antibody may correlate with protection. This property is important to consider potent vaccination strategies in addition to other factors such as the antigen payload [31]. In seronegative animals, maternally derived antibodies interfere with the response to FMD vaccines [32]. Vaccination is considered as main tool to prevent animals from FMD. But outbreaks of FMD have been reported after vaccination [10]. This was also observed in the findings that vaccinated animals infected $(0.94 \%)$ by the disease.

The finding also coincided with [11] who reported locally produced vaccines induce very low humoral immune responses against FMDV infection. The failure of vaccination may be due to differences between the circulating field strains and the strains included in the vaccine $[8,10]$. However, a shift of between FMDV serotypes may cause more severe outbreaks even in vaccinated animals. According to a study conducted by [8] cattle are at risk to FMD and cross bred are more susceptible. The vaccines are DIVA (differentiate infected from vaccinated animal) capable, to distinguish naturally infected and vaccinated animals. In the current study, FMD caused $0.5 \%$ mortality in young stock and and $0.43 \%$ in females. The overall fatality proportion in the vaccinated adult and young stock was $0.83 \%$ and $0.5 \%$ respectively.

This is in line with the findings of [33] who reported Foot-andMouth Disease caused high morbidity up to $100 \%$ and mortality $1 \%$ in calves and transmissibility is extremely high. In order to achieve better control of the disease in endemic areas, it is essential to monitor the current variants of the prevalent serotypes of FMDV in the field and ensure the most appropriate vaccine strains that are used to combat the circulating viruses and obtain knowledge about the origin of the outbreak. The potency of vaccine is the capacity of the vaccine to induce protective immunity and is expressed by PD50 for FMD virus [34]. Following post vaccination outbreak in the farms, the overall death of animals that manifested clinical signs was $0.9 \%$.

This because, the fast reduction in immunity post-vaccination leads susceptibility of cattle to infection. Animals required vaccines to develop and maintain adequate antibody levels when vaccinated at six months. Short-lived vaccine protection appears to have contributed to the high incidence of FMD. Vaccines provide better protection against challenge with homologous virus than antigenically different to the vaccine strains. Field viruses with poor vaccine match is responsible for disease incidence. Field studies of the vaccine during outbreaks of clinical disease is used to evaluate the effectiveness of vaccine and the potential of vaccination to reduce the risk in the vaccinated cattle against Foot and mouth disease. Although variation in the reactivity of sera against different viral strains reflect variation in the likelihood of protection. Quantification of protection requires virus challenge, either in the field or under controlled conditions, and for serological determination, protection must be correlated with a specific assay [29]. The challenge virus is homologous to the vaccine and test strain and protection in the field differs as the virus challenge is prolonged, although evaluation during field challenge is recommended [35-37].

\section{Conclusion and Recommendations}

Foot and mouth disease is a highly contagious acute vesicular viral disease that affects cloven-hoofed animals and is mainly controlled by vaccination. The circulation of the virus in susceptible livestock imposes severe restrictions on the movement and trade of animals and derived products, causing serious economic loss to the affected countries. In FMD endemic areas vaccination of susceptible populations is widely used as a major control measure. Commercial vaccine formulations that contain more than one virus strain is 
recommended to induce immune responses. Therefore, foot and mouth disease control should be instituted based on the availability of potent and master vaccines against the circulating field strains.

\section{References}

1. James A, Rushton J (2002) The economics of Foot and mouth disease. OIE scientific and Technical Rev 21(3): 637-644.

2. Paton DJ, Cox SJ, Voyce C, Parida S, Reid SM, et al. (2005) Protection against direct-contact challenge following emergency FMD vaccination of cattle and the effect on virus excretion from the oropharynx. Vaccine 23: 1106-1113.

3. Sahel M (2004) An epidemiological study on the genetic relationship on FMDV in East Africa. A thesis submitted in partial fulfillment for the degree of Doctor of Philosophy in veterinary tropical diseases, Faculty of Veterinary Science University of Pretoria, South Africa.

4. Ayelet G, Mahapatra M, Gelaye E, Egziabher G, Rufeal T, et al. (2009) Genetic characterization of foot-and-mouth disease viruses Ethiopia 1981-2007. J Emerg Infect Dis 15(9): 1409-1417.

5. Klein J (2009) Review on understanding the molecular epidemiology of foot and mouth disease virus. Infection, Genetics and Evolution 9: 153161.

6. Rweyemamu M, Roeder P, Mac Kay D, Leforban Y (2008) Planning for the progressive control of FMD worldwide. J Trans Emerg Dis 55: 73-87.

7. Kandeil A, ElShesheny R, Kayali G, Moatasim Y (2013) Characterization of the recent outbreak of FMD virus serotype SAT2 in Egypt. Arch Virol 158(3): 619-627.

8. Abubakar M, Khan, E Arshed MJ, Hussain M, Ali Q, et al. (2013) Mortality Rate in Association with FMD Outbreaks in Cattle, Pakistan. ASM Sci J 7(2): 139-143.

9. Bewket S, Mesfin S, Gelagay A (2012) Presentation on foot and mouth disease surveillance, laboratory and diagnostic activities in Ethiopia. East African Region Laboratory Network 3rd Annual Network Meeting, 5 March, Nairobi, Kenya.

10. Jamal SM, Ahmed S, Hussain M, Ali Q (2010) Status of foot-and-mouth disease in Pakistan. Arch Virol 155: 1487-1491.

11. Jamal SM, Shah SI, Ali Q Mehmood A, Dekker A (2013) Proper Quality Control of Formulated Foot-and-Mouth Disease Vaccines 61(6): 483489.

12. Yang M, Goolia MW, Bittner H (2013) Development of a quick and simple detection methodology for FMD virus serotypes $\mathrm{O}$, A and Asia 1 using a generic rapid assay devie. Virol J 10: 125.

13. Abubakar M, Jamil A, Ali Q (2014) An Evaluation of FMD Outbreak in Relation to Vaccination in a Herd of Cattle and Buffaloes. Res J vet Pract 2(2): 28-29.

14. Beksisa U (2017) Serotyping and molecular characterization of FMD virus isolated from outbreak cases in selected region and Addis Ababa, Ethiopia. MSc Thesis, Addis Ababa University College of Veterinary Medicine and agriculture, Bishoftu, Ethiopia.

15. (2009) CSA Central Statically Authority. Federal Democratic Republic of Ethiopia, Central Statically investigation, statically Abstract.

16. Jackson T (2002) Arginine glycine aspartic acid specific binding by FMD viruses to the purified integrin in vitro. J Virol 71(11): 8357-8361.

17. Admassu B, Getnet K, Shite A, Mohammed S (2015) Review on foot and mouth disease: Distribution and economic significance. Acad J Anim Dis $4: 160-169$
18. Ludi A, Mioulet V (2017) Laboratory diagnostic methods to support the surveillance and control of FMD. Foot and mouth dis. Virus, pp. 275.

19. Halloran ME, Ira Longini M, Struchiner CJ (1999) Design and interpretation of vaccine field studies. Epidemiologic reviews of Foot and mouth disease 21(1): 73-88.

20.Erdman DD, Heath JL, Watson Jc, Markowitz LE, Belini WJ (1993) Immunoglobin $\mathrm{M}$ antibody response to virus following primary and secondary vaccination and natural virus infection. Journal of Medical Virology 41: 44-48.

21. Halloran ME, Struchiner CJ (1995) Causal inference for infectious diseases. Epidemiology 89: 115-118.

22. Rodriguez LL (2009) Foot and mouth disease virus vaccines. vaccine 27: 90-94.

23. Halloran ME, Anderson RM, Azevedo neto, Bellini WJ, Branch O, et al. (1998) Population biology, evolution and immunology of vaccination programs. The American journal of Medical Sciences 315(5): 76-86.

24. Nair SP, K Sen (1992) A comparative study on the immune response of sheep to foot and mouth disease virus vaccine type Asia-1 prepared with different in activants and adjuvants Comp Immunol Microbi. Infect Dis 15: $117-124$.

25. Insel RA (1995) Potential alterations in immunogenicity by combining or simultaneously administering vaccine components. The New York Academy of Sciences 754: 35-47.

26. Dagan R, Eskola J, Leclerc C (1998) Reduced response to multiple vaccines sharing common protective epitopes that are administered simultaneously to infants. Infection and immunity 66(5): 2093-2098.

27. Harry guess A (1999) Combination vaccines: issues in evaluation of effectiveness and safety. Epidemiologic reviews 21(1): 89-95.

28. Robiolo B, Sergio Duffy, Emilio Leon, Nora Mattion (2010) Quantitative single serum-dilution liquid phase competitive blocking ELISA for the assessment of herd immunity and expected protection against FMD virus in vaccinated cattle, J Virol Methods 166(1-2): 21-27.

29. (2008) OIE. FMD, Manual of Diagnostic Tests and Vaccines for Terrestrial Animals, Version adopted by the World Assembly of Delegates of the OIE in May 2012, OIE Terrestrial, p. 1-29.

30. Arzt J, N Juleff, Z Zhang (2011) The pathogenesis of foot-and-mouth disease I: viral pathways in cattle. Trans-bound. Emerg Dis 58(4): 291304.

31. Cox SJ, Barnett PV (2009) Experimental evaluation of foot-and-mouth disease vaccines for emergency use in ruminants and pigs: a review. Vet Res, p. 40.

32. Patil CM, Sajjanar C (2014) Neutralizing antibody responses to FMD quadrivalent (type $\mathrm{O}, \mathrm{A}, \mathrm{C}$ and Asia 1 ) vaccines in growing calves with pre-existing maternal antibodies, Vet Microbiol, p. 5-7.

33. Mahy WJ (2004) Introduction and History of Foot-and-Mouth Disease Virus. Current Topics in Microbiology and Immunology 288: 1-8.

34. Doel TR (1996) Natural and vaccine-induced immunity to foot and mouth disease: the prospects for improved vaccines. Rev Sci tech Off Int Epiz 15(3): 883-911.

35. Knight Jones (2014) Retrospective evaluation of FMD vaccine effectiveness in Turkey. Vaccine 32: 1848-1855.

36. (2009) Veterinary world. Foot and mouth disease vaccination and postvaccination monitoring.

37. Abubakar M, Arshed MJ, Ali Q Hussain M (2012) Spatial Trend of FMDV Serotypes in Cattle and Buffaloes, Pakistan. Virolo Sinica 27(5): 320-323. 


\section{ISSN: 2574-1241}

DOI: 10.26717/BJSTR.2021.35.005685

Beksisa Urge. Biomed J Sci \& Tech Res

(c) (P) This work is licensed under Creative

Submission Link: https://biomedres.us/submit-manuscript.php

$\begin{array}{ll}\text { BIOMEDICAL } & \text { Assets of Publishing with us } \\ \text { RESEARCHES } & \text { Global archiving of articles } \\ \text { - Immediate, unrestricted online access }\end{array}$

